\title{
Adding Policy-based Control to Mobile Hosts Switching between Streaming Proxies
}

\author{
Malohat Ibrohimovna Kamilova ${ }^{1}$, Cristian Hesselman ${ }^{1,2}$, Ing Widya ${ }^{1}$, and Erik Huizer ${ }^{3}$ \\ ${ }^{1}$ CTIT, University of Twente, The Netherlands \\ ${ }^{2}$ Telematica Instituut, The Netherlands \\ ${ }^{3}$ Institute of Information and Computing Sciences, Utrecht University, The Netherlands \\ \{m.kamilova,i.a.widya\}@utwente.nl,cristian.hesselman@telin.nl,huizer@cs.uu.nl
}

\begin{abstract}
We add a simple policy-based control component to mobile hosts that enables them to control the continuous reception of live multimedia content (e.g. a TV broadcast) while they switch between different distributors of that content. Policy-based control provides a flexible means to automate the switching behavior of mobile hosts. The policies react to changes in the mobile host's environment (e.g. when a hotspot network appears) and determine when and how to invoke an earlier developed application-level protocol to discover the capabilities (e.g. supported encodings) of the content distributors and to execute the switches.

The design of the control component is based on the IETF policy model, but extended and applied at the application-level instead of at the network-level. We implemented the system and deployed it in a smallscale test bed.
\end{abstract}

\section{Introduction}

We consider the distribution of live multimedia content over the wireless Internet through multiple aggregators. An aggregator is an application-level redistributor that receives content from sources in the form of channels (e.g. CNN news) and forwards them to mobile hosts. Aggregators can deliver content in different configurations (e.g., in terms of price and quality), this enables them to serve different types of mobile hosts via a heterogeneous wireless network infrastructure. Figure 1 shows an example in which the roaming user Bob receives CNN news channel from media-forward.nl via UMTS provided by connect-it.nl at point A. At point B, Bob's mobile host detects the 802.11 provider hotspot.nl and switches via an 802.11 connection to a better aggregator of the channel (i.e. stream-it.com). At point C, Bob's mobile host switches back to the aggregator media-forward.nl available through UMTS.

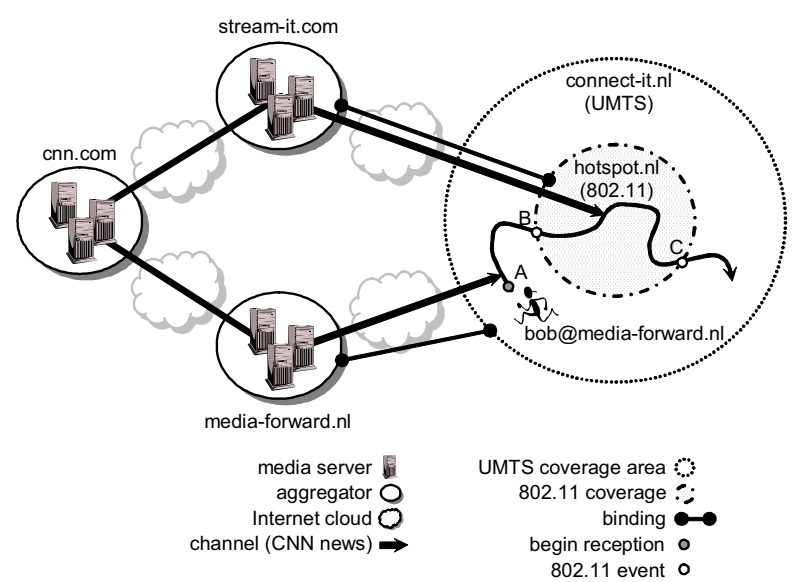

Figure 1. Streaming via multiple aggregators.

The research challenge addressed here is how to maintain the streaming of content despite the changes in the environment of the roaming mobile host or how to adapt the mobile host to the changes of the user's preferences. In this paper, we add a simple policybased control component to the mobile host that enables the host to automatically switch from one aggregator or configuration to another aggregator or configuration to receive or to continue to receive a content stream in a quality in accordance with the user's preference. A policy is basically an event, condition and actions construct [1-3]. In our work, the actions have to be executed by the mobile host if the specified events happen and the conditions are met (obligation policy).

We take a policy-based approach because it provides a flexible means to control when and how to discover and switch to the aggregator that offers the same channel at a quality or price that matches (better) with the user's preference. Moreover, the approach 
provides the capability to update the policies without interrupting the mobile hosts.

The next section describes the policy-based control system that runs on mobile hosts. The system contains the policies (which are briefly illustrated in Section 3) and it makes use of the earlier developed ALIVE protocol [4], discussed in Section 4. The ALIVE protocol enables mobile hosts to discover the available configurations of a particular channel at reachable aggregators and to switch from one of the aggregators to another. Section 5 describes the testbed in which we validate our implementation. Thereafter, we discuss related work and in Section 7, we present our conclusions and future work.

\section{Policy-controlled Switching}

Figure 2 shows the architecture of the ALIVE control system. It is inspired by the IETF policy model $[1,2]$ for network management, but applied at the application-level for switching control. The main components of the architecture are the ALIVE controller (which includes the IETF Policy Decision Point (PDP)) and the ALIVE client protocol entity (basically, the Policy Enforcement Point (PEP)).

The other components are:

- a policy repository for storing ALIVE policies;

- an environment monitor that detects environment changes (e.g. the appearance of a new network) and informs the ALIVE controller PDP of such changes;

- a user preferences manager that allows the user to specify his preferences, for instance a preference for the cheapest configuration or a smooth play-out without glitches. This manager also informs the ALIVE controller PDP of changes in the user's preferences (e.g. when new preferences lead to another criterion of what is considered as 'best' configuration).

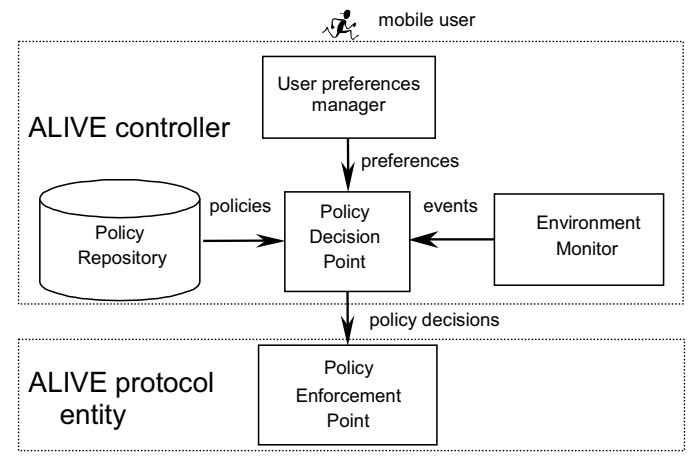

Figure 2. ALIVE control system's architecture.

The ALIVE PDP encapsulates a set of policies that control the switching behavior of the mobile host. It is event-driven and evaluates its policies using the information provided by the preferences manager or the environment monitor. The policy decisions of the ALIVE PDP are either "discover" or "switch", which are operations provided by the ALIVE protocol entity to initiate configuration discovery or to initiate a switch. A discovery decision also specifies the maximum length of the collection period for accepting discovered aggregators or configurations, before the ALIVE controller PDP decides on the need for a switch to another aggregator or configuration. Besides a description of the decided best configuration, a switching decision also contains the order in which the protocol entity should execute the switch (i.e. breakbefore-make or make-before-break).

\section{ALIVE Policies}

Figure 3 illustrates an ALIVE discovery policy expressed in XML. ALIVE policies have an operational type (i.e. discover or switch) and a goal (e.g. expressing a highly smooth switching strategy). The illustrated policy uses the highly smooth switching strategy, which initiates discovery when the average packet loss increases beyond $20 \%$. Hysteresis and back-off techniques in cost-benefit conditions can be further used to resolve the switching back and forth between aggregators along edges of wireless networks.

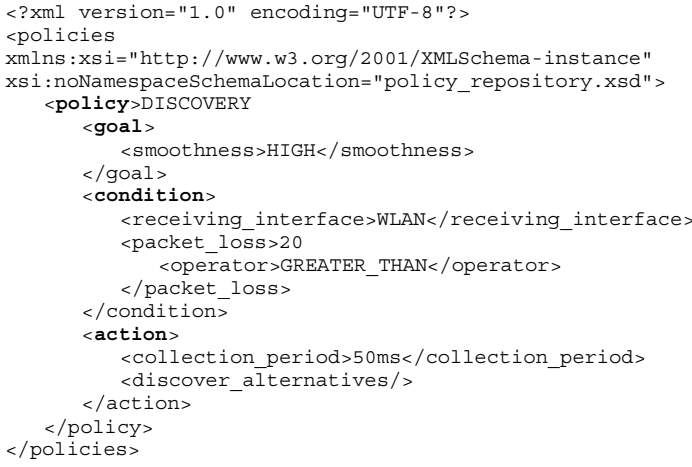

Figure 3. Discovery policy in XML.

Most policies are stored in the policy repository. Other policies residing in the ALIVE controller may trigger the downloads of some of the policies in the repository at run-time (i.e. without halting the mobile host). Policy downloads flexibly change the switching behavior of mobile hosts.

In general, a PDP can download policies using complex criteria under predefined circumstances. The ALIVE PDP downloads policies based on their goals (e.g. retrieving policies that realize a highly smooth switching strategy). We further simplify policy downloads by expressing the user preferences in the same terms as the policy goals. This means that the 
user preferences directly determine which policies the PDP will download. For example, if the user does not mind perceptual glitches, then the ALIVE controller will for instance download policies that realize a moderately smooth switching strategy (both discovery and switching policies). This example also shows that a change of user preferences can trigger the PDP to download new policies from the policy repository.

\section{Behavior}

Figure 4 shows a typical behavior of the ALIVE controller, e.g. at point B in Figure 1.

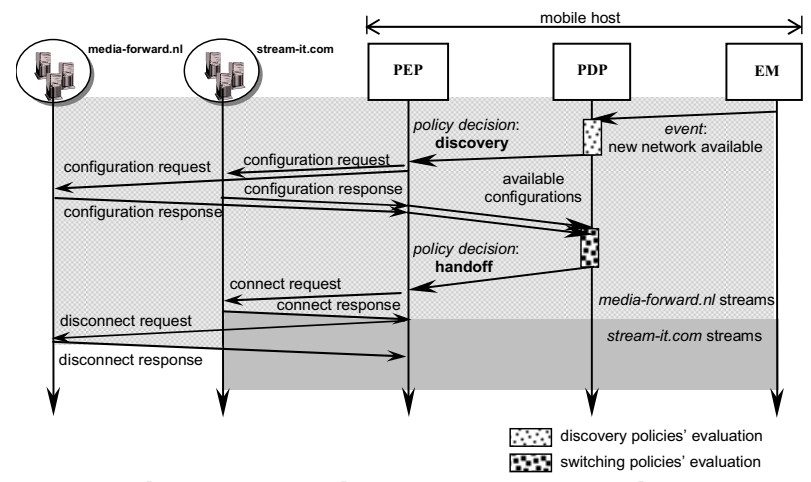

Figure 4. Typical system behavior.

At this point, the environment monitor (EM) detects the availability of the 802.11 network of hotspot.nl and reports this event to the PDP. The event triggers the evaluation of the discovery policies in the PDP. In this example, the result is that the PDP decides to instruct the ALIVE protocol entity to transmit configuration requests to stream-it.com and media-forward.nl. The PEP passes the configuration descriptions in the configuration responses back to the PDP. The PDP's switching policies then evaluate the responses and decide that stream-it.com provides $\mathrm{CNN}$ news in the best configuration and control the mobile host to switch in a make-before-break manner (which e.g. is a part of the highly smooth switching strategy). The PEP first connects the mobile host to stream-it.com and then disconnects it from media-forward.nl. As a result, the mobile host receives CNN news from stream-it.com via the 802.11 network of hotspot.nl.

\section{Testbed}

We have implemented ALIVE controller in C. A simple user interface for providing user preferences is implemented in Tcl/Tk. As a component of the EM we use the Mobility Manager [5], which keeps track of the changes on the network interfaces of the mobile host. We deployed our implementation in a small-scale testbed. The idea behind the setup is to emulate the scenario of Figure 1. Since we did not have access to a wide-area wireless network that could carry video streams (typically UMTS), we used an 802.11 wireless LAN to emulate the wide area network and an Ethernet to emulate the hotspot. This enabled us to emulate roaming by plugging the Ethernet cable in and out of the mobile host.

Figure 5 shows the set up. The main components are a laptop, a server, an 802.11 access point and a fixed Ethernet. The laptop represents the mobile host of Figure 1. It is equipped with an Orinoco 802.11b Gold card and an Ethernet card. The 802.11 base station and the Ethernet LAN form two separate subnets representing two separate network operators. The server hosts the two aggregator domains of Figure 1 in the form of two processes (see below).

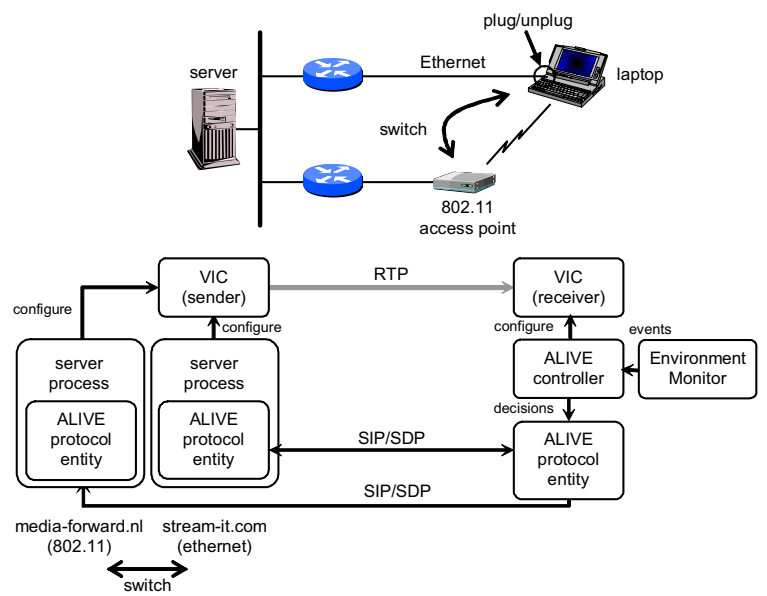

Figure 5. ALIVE testbed

The ALIVE controller and client protocol entity run on the laptop, which also runs VIC [6] in receiver mode to receive and render RTP multimedia packets. The ALIVE protocol builds on OpenSIP [7], a C implementation of the Session Initiation Protocol (SIP) that also includes support for configuration descriptions through the Session Description Protocol (SDP). The server runs two processes that execute the server-side of the ALIVE protocol. One process represents the aggregator media-forward.nl, the another stream-it.com. The server runs the VIC in the sender mode to emulate the streaming servers of the two aggregators. We have extended the VIC server so that it also can interpret configuration descriptions and use that information to configure VIC's framerate, quality, and bitrate (kbps). The extension can also dynamically configure VIC to stream to another IP address, for instance to the IP address of the laptop's Ethernet interface instead of to its 802.11 interface. 
As an example scenario, assume that the laptop receives a multimedia stream from 'media-forward.nl' via the 802.11 'overlay network'. If we plug in the Ethernet cable (i.e. enter the 'hotspot'), the ALIVE controller will evaluate triggered policies and will decide to run the discovery part of the ALIVE protocol with a collection period of $50 \mathrm{~ms}$ for a highly smooth switching strategy or 1 second for a moderate strategy.

In the collection period, the ALIVE controller collects the responses that the ALIVE protocol entity receives from 'media-forward.nl' and 'stream-it.com'. At the end of this period or earlier if all aggregators have responded, the ALIVE controller PDP decides to switch the mobile host to stream-it.com. Therefore it informs the ALIVE protocol of the decision, which then sends a connect message to stream-it.com. This connect message includes a description of one of stream-it.com's configurations (the best one).

After the switch, the laptop receives the channel from stream-it.com via its Ethernet interface. The configuration in which it will receive the channel will typically be of a higher quality because the Ethernet has a higher capacity than the 802.11 network.

Figure 6 presents screenshots of the laptop before and after the switch. The window at the bottom shows a few status lines of the controller during the switch.

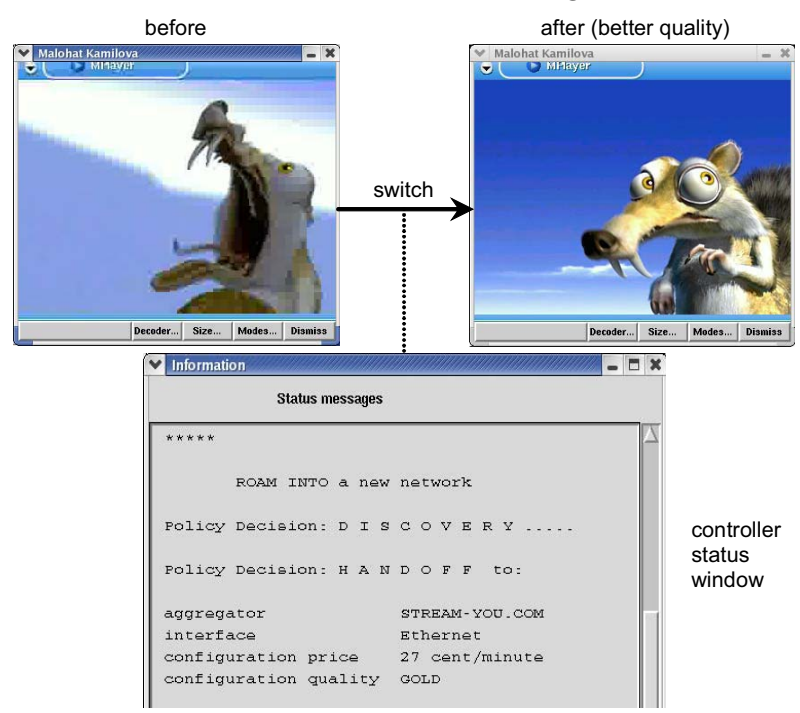

Figure 6. Screenshots illustrations.

\section{Related work}

Many of the known policy-based systems for Internet service control typically use network level policies rather than application-level policies and they focus on determining which network (operator) provides the best service [8-10]. Wang et al. [8] consider policy-enabled handoffs between different networks, but do not consider switches between application level content providers. Furthermore, their policies are based on user preferences (e.g. price, power consumption and quality), while our policies are also based on changes in the environment (e.g., packet losses and signal strength). Murray et al. [9] discuss the selection of a best network for a mobile host according to the current load on the networks. The selection in their system is controlled by policy decision logic that sits in the infrastructure, while ours sits on mobile hosts. Lee et al. [10] take a completely different approach to determine the best network service, they use predictors rather than policies.

\section{Conclusions}

In this paper, we proposed a policy-based switching control component that runs on top of an existing application-level protocol. The prototype implementation demonstrates the flexibility of policybased control. Further, we have shown the viability of applying the IETF's policy model at the application level for controlling the switching behavior of the mobile host across alternative aggregators.

Current ongoing work is on measuring the performance of the chosen protocol stack to enable policy condition fine tuning such as the detection of cell edges, etc. Future work constitutes the deployment of new policies and the addressing of policy conflicts.

\section{References}

[1] RFC 2753, A Framework for Policy-based Admission Control, January 2000.

[2] RFC 3060, Policy Core Information Model, 2001.

[3] M. Cox and R. Davison, "Concepts, Activities and Issues of Policy-based Communications Management", BT Technology Journal, V 17, Issue 3, July 1999, pp. 155-169.

[4] C. Hesselman, H. Eertink, I. Widya, and E. Huizer, "Delivering Live Multimedia Streams to Mobile Hosts in a Wireless Internet with Multiple Content Aggregators", to appear in Mobile Networks and Applications journal (Kluwer Wireless), Summer 2005.

[5] A. Peddemors, H. Zandbelt, and M. Bargh, "A Mechanism for Host Mobility Management supporting Application Awareness ", In Proc. MobiSys'04, June 2004.

[6] VIC, http://www-mice.cs.ucl.ac.uk/multimedia/software/ [7] oSIP webpage, http://www.gnu.org/software/osip/

[8] H.Wang, R. Katz, J.Giese, "Policy-Enabled Handoffs Across Heterogeneous Wireless Networks", In Proc. WMCSA 1999, New Orleans, USA, February 1999.

[9] K. Murray, R. Mathur, D. Pesch, "Intelligent Access and Mobility Management in Heterogeneous Wireless Networks using Policy", In Proc. The $1^{\text {st }}$ IS on ICT, Dublin, 2003.

[10] G. Lee, P. Faratin, S, Bauer and J. Wroslawski, "Automatic Service Selection in Dynamic Wireless Network Environments", poster MobiCom'03, SanDiego, USA, 2003. 